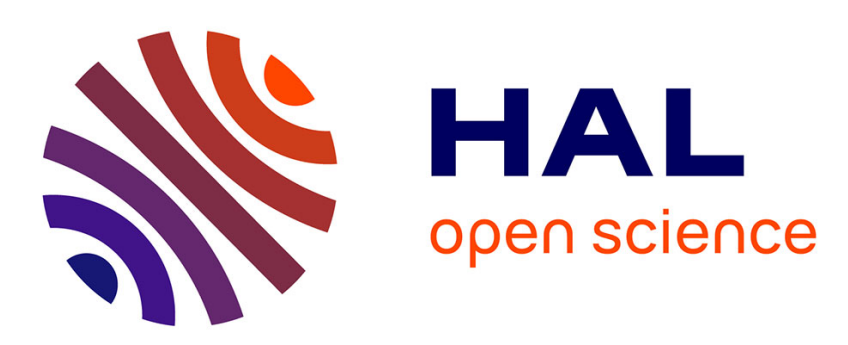

\title{
Efficient modeling approach for optimization of a system based on passive diamagnetic levitation as a platform for bio-medical applications"
}

Hichem Chetouani, Benoît Delinchant, Gilbert Reyne

\section{To cite this version:}

Hichem Chetouani, Benoît Delinchant, Gilbert Reyne. Efficient modeling approach for optimization of a system based on passive diamagnetic levitation as a platform for bio-medical applications". COMPEL: The International Journal for Computation and Mathematics in Electrical and Electronic Engineering, 2007, 26 (2), pp.345 - 355. hal-00334017

\section{HAL Id: hal-00334017 https://hal.science/hal-00334017}

Submitted on 21 Jan 2019

HAL is a multi-disciplinary open access archive for the deposit and dissemination of scientific research documents, whether they are published or not. The documents may come from teaching and research institutions in France or abroad, or from public or private research centers.
L'archive ouverte pluridisciplinaire HAL, est destinée au dépôt et à la diffusion de documents scientifiques de niveau recherche, publiés ou non, émanant des établissements d'enseignement et de recherche français ou étrangers, des laboratoires publics ou privés. 


\title{
Efficient modeling approach for optimization of a system based on passive diamagnetic levitation as a platform for bio-medical applications
}

\author{
H. Chetouani, B. Delinchant, G. Reyne \\ Laboratoire d'Electrotechnique de Grenoble (LEG) \\ ENSIEG, Domaine Universitaire, BP 46, \\ F-38402 Saint Martin d'Hères Cedex, FRANCE
}

\begin{abstract}
Constrained optimization may use time-consuming analysis on standard methods such as the finite-element method (FEM). In order to decrease the optimization time and to enable flexible gradient based optimization, this paper reports, a semi-analytical modelling approach and optimization of two variants of a diamagnetic levitation system as contactless manipulation platform for bio-medical and biochemical applications.
\end{abstract}

Key words: Optimal design, Bio-medical systems, Microsystems, Diamagnetic levitation.

\section{LEVITATION IN BIOMEDICAL APPLICATIONS}

The magnetic biochips are in increasing development and evolution supported by several applications in the biochemical and biomedical fields [1-4]. This family of microsystems includes partial or complete integrations of elementary or complex tasks processed by traditional laboratories of our scale. The automation and parallelization of these tasks [2] in the small world avoids human handling which is frequently not precise and likely of errors, and reduces times and the costs necessary to achieve operations such as diagnostic, analyzes and particles sorting [2-4]. This evolution of micro-actuators based on magnetic actuation has lead to an increasing need for specific modeling methodologies especially for optimization requirements. Thus, in order to facilitate the use of gradient based optimization process [5], this work deals with a realistic semi-numerical modeling approach of a system based diamagnetic levitation declined in two variants. Indeed, these systems have more and more applications, especially in biomedical field [6-7]. Diamagnetic contactless handling of bioparticles brings amazing perspectives to biochips and avoids serious problems as adhesion, adsorption and contamination.

\section{MAGNETOPHORESIS FORMULATION AND MODELING}

Currently, existing modeling approaches treating diamagnetic levitation problems are numerical methods such as finite elements method or magnetic moments method [8]. Theses modeling approaches are not adequate for full design studies. Therefore, in order to permit an adaptable optimization with gradient based algorithms, semianalytical modeling was chosen and then established to compute outputs and formally exact sensitivities according to the inputs parameters of design [9]. The proposed device comprises four fix magnets and a levitating diamagnetic object. In order to approach both possible diamagnetic materials: anisotropic and isotropic and both possible fluids for biological bodies: air and liquid, the models of a levitating anisotropic graphite plate in air, and a levitating isotropic latex sphere in fluid were established. The magnetic field generated by these four parallelepiped magnets was expressed using equivalent surface method [10]. In this formulation the induced induction of the diamagnetic levitating object was neglected. This induced field did not affect significantly the external field due to the low magnetic susceptibilities of the diamagnetic materials. Hereafter is reported the magnetophoretic force [11] formulations corresponding to both proposed cases.

\section{A. Modeling of an anisotropic diamagnetic graphite plate in air}

A diamagnetic anisotropic plate in air with $(2 \mathrm{a}, 2 \mathrm{~b}, 2 \mathrm{c})$ dimensions, having magnetic susceptibilities $\left(\chi_{\mathrm{x}}, \chi_{\mathrm{y}}, \chi_{\mathrm{z}}\right)$ respectively on $\mathrm{x}, \mathrm{y}$ and $\mathrm{z}$-axis and within a magnetic field induction expressed by its components $\mathrm{B}_{\mathrm{x}}, \mathrm{B}_{\mathrm{y}}$ and $\mathrm{B}_{\mathrm{z}}$, experiences a magnetic force obtained from integral over whole diamagnetic plate volume (2). According to Ostrogradsky theorem (3) [12], magnetic force components (magFx, magFy and magFz) can be simplified leading to surface integrals (4-6). This simplification allows to establish a flexible semi-numerical model based on surface integrals to compute the magnetic force components and thus to enables a faster optimization.

$$
G(x, y, z)=\chi x \cdot B x(x, y, z)^{2}+\chi y \cdot B y(x, y, z)^{2}+\chi z \cdot B z(x, y, z)^{2}
$$




$$
\begin{gathered}
\operatorname{mag} F(x, y, z)=\frac{1}{2 \mu_{0}} \iiint_{v} \nabla G(x, y, z) \cdot d v \\
\iiint_{v} \nabla f \cdot d v=\oiint \int_{s} f \cdot \vec{n} \cdot d s \\
\operatorname{mag} F x(x, y, z)=\frac{1}{2 \mu_{0}} \iiint_{v} \frac{\partial G(x, y, z)}{\partial x} \cdot d v=\frac{1}{2 \mu_{0}} \int_{-c-b}^{c} \int^{b}(G(x+a, y+Y, z+Z)-G(x-a, y+Y, z+Z)) \cdot d Y . d Z \\
\operatorname{mag} F y(x, y, z)=\frac{1}{2 \mu_{0}} \iiint_{v} \frac{\partial G(x, y, z)}{\partial y} \cdot d v=\frac{1}{2 \mu_{0}} \int_{-c-a}^{c} \int_{-a}^{a}(G(x+X, y+b, z+Z)-G(x+X, y-b, z+Z)) \cdot d X . d Z \\
\operatorname{mag} F z(x, y, z)=\frac{1}{2 \mu_{0}} \iiint_{v} \frac{\partial G(x, y, z)}{\partial z} \cdot d v=\frac{1}{2 \mu_{0}} \int_{-b-a}^{b} \int^{a}(G(x+X, y+Y, z+c)-G(x+X, y+Y, z-c)) \cdot d X . d Y
\end{gathered}
$$

where $\mu_{0}$ is the vacuum magnetic permeability.

\section{B. Modeling of an isotropic diamagnetic latex sphere in a liquid}

A diamagnetic isotropic sphere having a radius $\mathrm{R}$ within a susceptible liquid experiences a magnetic force according to magnetic susceptibility contrast $\Delta \chi$ between the liquid and the sphere material. This force is obtained with an integral over the whole sphere volume (9). Then magnetic force components (magFx, magFy and $\mathrm{magFz}$ ) can be simplified leading to surface integrals (10-12). This simplification allows to establish a flexible semi-numerical model to compute magnetic force components based on surface integrals and thus a faster optimization.

$$
\begin{aligned}
& G(x, y, z)=B(x, y, z)^{2}=B x(x, y, z)^{2}+B y(x, y, z)^{2}+B z(x, y, z)^{2} \\
& g 1(y, z)=\sqrt{R^{2}-y^{2}-z^{2}}, g 2(x, z)=\sqrt{R^{2}-x^{2}-z^{2}}, g 3(x, y)=\sqrt{R^{2}-x^{2}-y^{2}} \\
& \operatorname{mag} F(x, y, z)=\frac{\nabla \chi}{2 \mu 0} \iiint \nabla G(x, y, z) \cdot d v \\
& \operatorname{mag} F x(x, y, z)=\frac{\nabla \chi}{2 \mu_{0}} \int_{-R_{-} \sqrt{R^{2}-Y^{2}}}^{R} \int_{R^{2}-Y^{2}}(G(x+g 1(Y, Z), y+Y, z+Z)-G(x-g 1(Y, Z), y+Y, z+Z)) \cdot d Z . d Y \\
& \operatorname{mag} F y(x, y, z)=\frac{\nabla \chi}{2 \mu_{0}} \int_{-R_{-} \sqrt{R^{2}-Z^{2}}}^{R} \int_{R^{2}-Z^{2}}(G(x+X, y+g 2(X, Z), z+Z)-G(x+X, y-g 2(X, Z), z+Z)) \cdot d X . d Z \\
& \operatorname{mag} F z(x, y, z)=\frac{\nabla \chi}{2 \mu_{0}} \int_{-R_{-} \sqrt{R^{2}-X^{2}}}^{R} \int_{R^{2}-X^{2}}(G(x+X, y+Y, z+g 3(X, Y))-G(x+X, y+Y, z-g 3(X, Y))) \cdot d Y . d X
\end{aligned}
$$

\section{Automated modeling tool}

A modeling tool [13] based on this diamagnetic computation method was developed. Designer has to describe the geometrical and physical system (size, magnetic parameters, etc) and the tool generates automatically these formulas. This tool (Fig. 1) computes forces with magnets, current conductors with several shapes (parallelepiped, sphere, cylinder, arc of cylinder, etc).

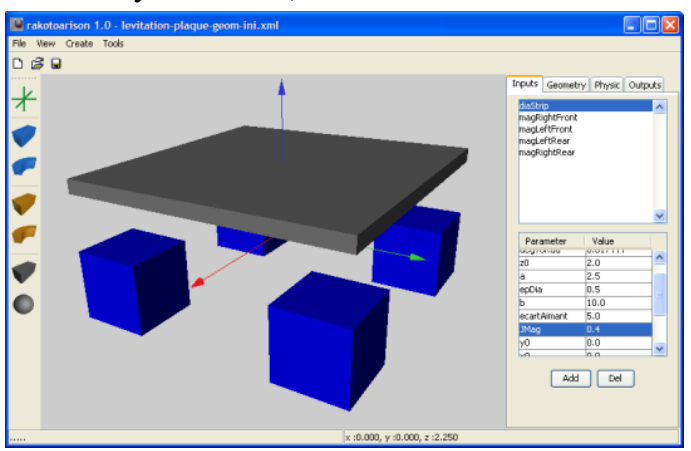

Fig. 1 - Interface screenshot of the tool used to generate designs computation formulas.

\section{MODELING VALIDATION}

The proposed device is composed of a diamagnetic anisotropic plate having a susceptibility $\chi_{z}=-4.510^{-4}$ and $\chi_{x}=$ $\chi_{y}=-8.10^{-5}$, a density $\rho_{\mathrm{g}}=2200 \mathrm{~kg} / \mathrm{m}^{3}$. It levitates above four magnets configured as shown on Fig. 2-(a). The magnetic vertical force experienced by the plate was computed semi-analytically, and then it was compared to the magnetic moment method simulation for which the induced magnetization of diamagnetic object is not neglected. The results obtained from these two modeling methods are reported on Fig. 2-(b). These results show 
a performing modeling and that approximation (no induced induction into the diamagnetic material) is realistic.

(a)

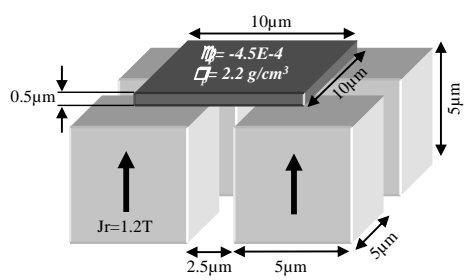

(b)

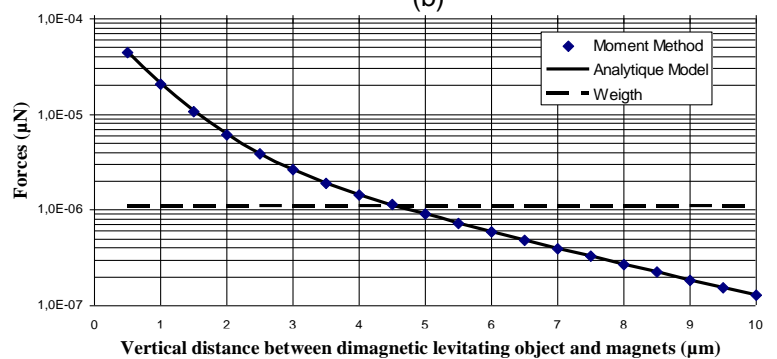

Fig. 2 - (a) Device configuration, (b) Simulation results of the magnetic force Fz obtained from the semianalytical model and the magnetic moment method.

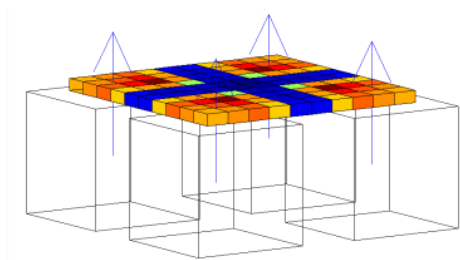

Fig. 3 - The design meshed with an tool based magnetic moments computation.

FEM computation was also compared with these modeling techniques but has lead to interpretation issues. Indeed, diamagnetic forces, which are about some picoNewtons, are drowned into meshing noise. Thus to compute forces with a FEM tool, meshing noise has to be removed by this technique.

- Compute the force on the diamagnetic plate with $\chi=0$. The force value result only on the noise.

- Compute the force on the diamagnetic plate with real $\chi$ value, without changing the meshes.

- $\quad$ Substract the result value with preceding value to obtain the real force (Table. 1).

TABLE I: FORCE COMPUTATION WITH FEM METHOD

\begin{tabular}{|l|l|l|l|}
\hline Vertical distance $(\mu \mathrm{m})$ & $\begin{array}{c}\text { Force computation with } \\
\chi=0(1)\end{array}$ & $\begin{array}{c}\text { Computation with } \\
\chi=10^{-5} \times[-8,-8,4.5](2)\end{array}$ & $\begin{array}{c}\text { Resulting } \\
\text { force }=(2)-(1)\end{array}$ \\
\hline 3 & $13.5725018 \mathrm{E}-9$ & $13.5698702 \mathrm{E}-9$ & $2.6316 \mathrm{E}-12$ \\
\hline
\end{tabular}

\section{OPTIMIZATION}

The optimizations performed tries to converge towards magnets structures for given levitating objects and levitation heights. Its objective is to maximise the spatial stability of the object. The stability of the levitating object is quantified by the total force gradient acting on corresponding axis. These optimizations respond to precise constrained specifications. A semi-analytical model characterizing the device was established. It enables computation of the forces exerted on the diamagnetic levitating object and theirs corresponding gradients as outputs. These outputs are required to express the stability of the levitating object according to structure geometry and physical parameters. In order to use an efficient optimization algorithm like SQP (Sequential Quadratic Programming), an automatic tool was used to generate formally exact sensitivities of the established semi-analytical model. This tool is called a generator and is part of the CADES design framework pattern shown Fig. 4, which describes how model and optimizer can interact in an iterative and reusable manner.

(a)

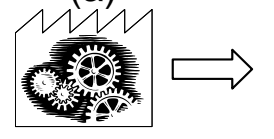

Fig. 4 - (a) Generators create, (b) components, (c) Services use.

(c)
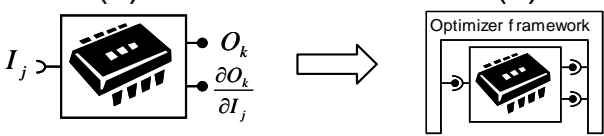

The symbolic derivation of the model produces formal exact sensitivity (without noise like finite differences) but also quicker model. Indeed, force sensitivity depending on a diamagnetic size parameter, leads to a simple integral formula as describe in the following equations. Thus, as numerical integration take most of the model computation time, this may reduce consequently the global sizing time. 


$$
\begin{gathered}
f(a, b)=\int_{0}^{b} \int_{0}^{a} g(x, y) d x d y \\
\frac{\partial f(a, b)}{\partial b}=\int_{0}^{a}[g(x, b)-g(x, 0)] d x
\end{gathered}
$$

Using this design framework, the graphite plate and the polystyrene latex sphere devices were sized as shown in the following.

\section{A. Diamagnetic anisotropic graphite plate in air}

The device comprises a fixed part and a mobile one as shown on Fig. 5. The fix part of the device comprises four electroplated CoPt magnets [14] magnetized throughout the plane with a residual magnetization $\mathrm{Jz}=0.3 \mathrm{~T}$. These magnets are identical and have as parameters magsize, magheight and gap which correspond respectively to the magnets squared base size, magnets height and distance between two adjacent magnets. The mobile part is a plate made of pyrolitic graphite which is an anisotropic material. Its magnetic susceptibilities on $\mathrm{x}, \mathrm{y}$ and $\mathrm{z}$ axis are $\left[\chi_{x}, \chi_{y}, \chi_{z}\right]=10^{-5} \times[-8,-8,-45]$ and its density $\rho_{\mathrm{g}}=2200 \mathrm{~kg} / \mathrm{m}^{3}$. This plate has as parameters: diasize (the squared base size of the plate) and diaheight (height of the plate). Both parameters were fixed respectively at 10 $\mu \mathrm{m}$ and at $0.5 \mu \mathrm{m}$. The objective of optimization was to converge towards a solution giving an optimal stability satisfying a fixed levitation height of $2 \mu \mathrm{m}$. This levitation height is computed between top of magnets and bottom of diamagnetic plate at position where the total force on $\mathrm{z}$ axis become null. Neglecting the Archimedes force due to low density of the medium. Only two forces act vertically on the diamagnetic plate: the weight of the plate (diaweight) and the magnetic force (magFz) (6). Therefore, the balance of vertical forces FtotZ can be expressed as:

$$
\text { Ftot } Z=m a g \vec{F} z+\text { diaWeight }
$$

The parameters magsize, magheight and gap were constrained as given on Table. 2. The optimization results corresponding to these constrained specifications are illustrated on Table. 3, where is given inputs parameters and the computed outputs values corresponding to two geometries. The diamagnetic plate at the first geometry shown on Fig. 5-(a) has a levitation height of $1.75 \mu \mathrm{m}$ and is unstable on $\mathrm{x}$ axis and stable on vertical one. The second geometry shown on Fig. 5-(c) corresponds to an optimal stability obtained after optimization. The forces curves corresponding to both devices are shown on Fig. 6.

TABLE II: OPTIMIZATION SPECIFICATIONS

\begin{tabular}{|c|c|c|c|c|}
\hline Constrained parameters $(\mu \mathrm{m})$ & magsize $=[1,20]$ & magheight $=[1,10]$ & gap $=[0.1,5]$ & Dist=2 \\
\hline Objective & \multicolumn{3}{|c|}{ Optimal stability } \\
\hline
\end{tabular}
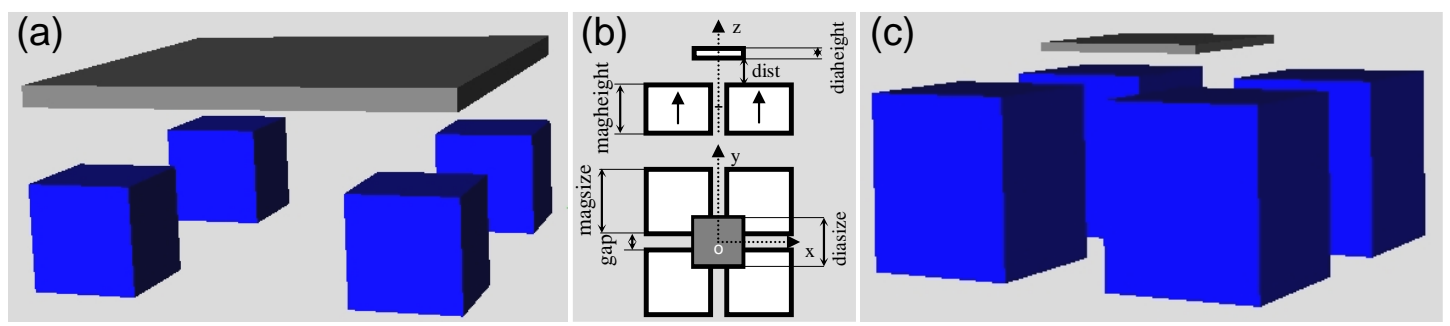

Fig. 5 - (a) Geometry of device with unstable plate on x axis, (b) Geometry parameters of the diamagnetic plate and the magnets, (c) Optimal design for axial stability of the diamagnetic anisotropic plate.

TABLE III: UNSTABLE DESIGN PARAMETERS AND OPTIMIZATION RESULTS

\begin{tabular}{|l|l|l|l|l|}
\hline \multicolumn{1}{|c|}{ Name } & \multicolumn{1}{c|}{ Designation } & \multicolumn{1}{c|}{ Unstable design } & \multicolumn{1}{c|}{ Optimal design } & unity \\
\hline diaWeight & Weight of diamagnetic plate & -1.0788 & -1.0788 & $\mathrm{pN}$ \\
\hline magFZ & Vertical magnetic force & -1.0799 & 1.0791 & $\mathrm{pN}$ \\
\hline FtotZ & Balance of vertical forces & $1.1611 \mathrm{E}-9(\sim 0 \mathrm{~N})$ & $3.0364 \mathrm{E}-10(\sim 0 \mathrm{~N})$ & $\mu \mathrm{N}$ \\
\hline gradFx_x & Gradient of Fx along $x$ axis & $0.1808($ unstable $)$ & $-1.3668($ stable $)$ & $\mu \mathrm{N} / \mathrm{m}$ \\
\hline gradFz_z & Gradient of Fz along z axis & $-0.1916($ stable $)$ & $-1.672($ stable $)$ & $\mu \mathrm{N} / \mathrm{m}$ \\
\hline gap & Distance between two magnets & 5 & 6.4430 & $\mu \mathrm{m}$ \\
\hline magheight & Magnets height & 2.5 & 10 & $\mu \mathrm{m}$ \\
\hline magsize & Magnets squared base size & 2.5 & 8.5960 & $\mu \mathrm{m}$ \\
\hline
\end{tabular}



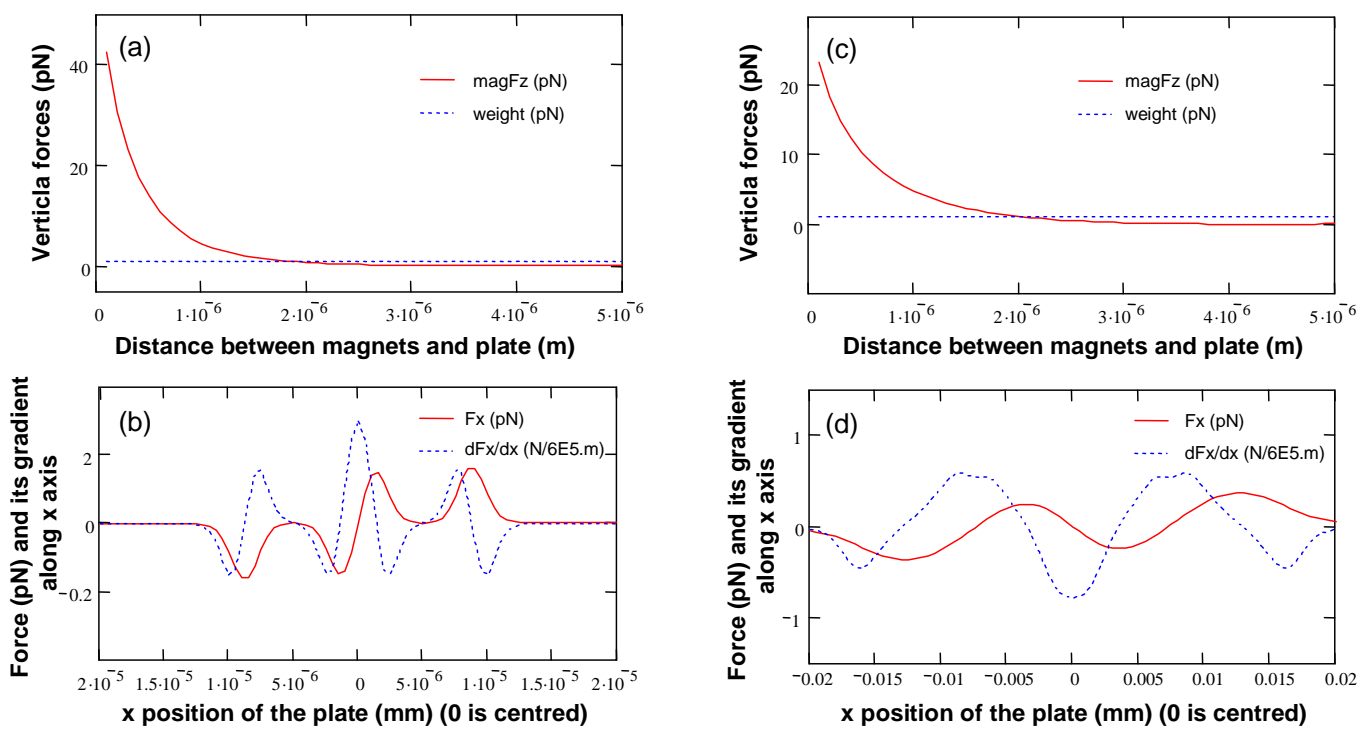

Fig. 6 - Forces experienced by the diamagnetic plate, (a) vertical forces according to the distance between the magnets and the plate at the case of the unstable design, (b) horizontal force and its gradient according to the $\mathrm{x}$ position of the plate at the case of the unstable design, (c, d) Case of the stable design.

The Pareto optimization results shown on Fig. 7 validate the obtained results of the classic optimization. This one was done in two steps. Firstly isovalues of the gradient of the force on $x$ axis where plotted as function of the parameters gap and magsize. Secondly the curve of the parameter gap was plotted as a function of magsize with the total force on the vertical axis and its gradient constrained to be respectively null and negative to satisfy the stability criterion on vertical axis, where the force on $\mathrm{x}$-axis and its gradient were free.

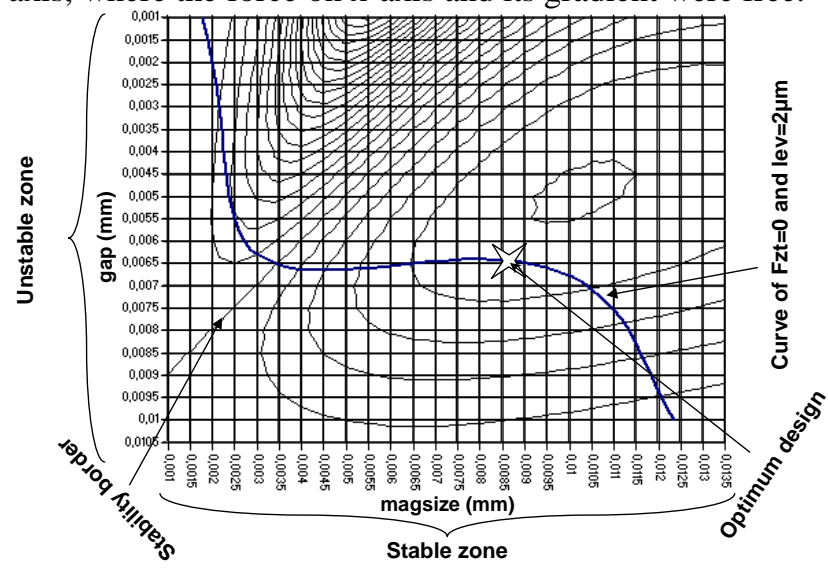

Fig. $7-2 \mathrm{D}$ cartography of stability on $\mathrm{x}$ axis and the optimal design localisation expressed according to the squared base size of the magnet and the gap between the magnets.

\section{B. Diamagnetic latex bead in paramagnetic medium}

The fix part of device is still the same used above for the diamagnetic graphite plate in air. However the mobile part is now a diamagnetic sphere made of polystyrene latex which is an isotropic material. Its susceptibility is $\chi_{\mathrm{p}}=-8.2 \cdot 10^{-6}$ and its density $\rho_{\mathrm{p}}=1050 \mathrm{~kg} / \mathrm{m}^{3}$. The medium is a paramagnetic configured with about $0.47 \mathrm{M}$ of manganese chloride tetrahydrate $(\mathrm{MnCl} 2.4 \mathrm{H} 2 \mathrm{O})$ in pure water, having a magnetic susceptibility $\chi_{\mathrm{m}}=7.10^{-5}$ and a density $\rho_{\mathrm{m}}=1000 \mathrm{~kg} / \mathrm{m}^{3}$. This sphere has as parameter diasize (the radius of the sphere) fixed at $1.5 \mu \mathrm{m}$. The objective of optimization is to converge towards a solution giving an optimal stability satisfying a levitation height of $3 \mu \mathrm{m}$. The parameters magsize, magheight and gap were constrained as given on Table. 4. The optimization results corresponding to these constrained specifications are shown on Table. 5. It illustrates the inputs parameters and the computed outputs values corresponding to two geometries. The diamagnetic sphere is unstable at the first geometry shown on Fig. 8-(a) on $\mathrm{x}$ axis and stable on vertical one and the levitation height is $3 \mu \mathrm{m}$. The second one shown on Fig. 8-(c) corresponds to a solution giving an optimal horizontal stability which was obtained after optimization process. The levitation height is computed between top of magnets and bottom of diamagnetic sphere at the position where the total force on $\mathrm{z}$ axis become null. The levitation height is fixed at 
dist $=3 \mu \mathrm{m}$. This position is determined from the balance of forces experienced by the sphere on vertical axis. Due to spherical geometry and the density of the medium, three forces acts verticaly: diaweight, diaArch (16) and FtotZ (17) respectively the weight of the sphere, the Archimedes force and the balance of forces. The forces curves corresponding to unstable and stable devices are shown on Fig. 9.

$$
\text { diaArch }=\frac{4}{3} \pi\left(\frac{\text { diasize }^{3}}{8}\right) \rho_{m} g
$$

Ftot $Z=m a g \vec{F} z+\operatorname{diaWeight}+\operatorname{diaA} r c h$

\section{TABLE IV: OPTIMIZATION SPECIFICATIONS}

\begin{tabular}{|c|c|c|c|c|}
\hline Constrained parameters $(\mu \mathrm{m})$ & magsize $=[4,12]$ & magheight $=[4,10]$ & gap $=[1,7]$ & Dist $=3$ \\
\hline Objective & \multicolumn{5}{|c|}{ Optimal stability on $\mathrm{x}$ axis } \\
\hline
\end{tabular}
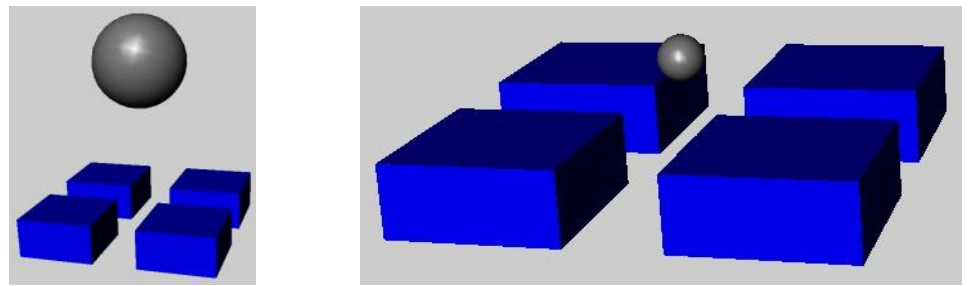

Fig. 8 - (a) Geometry of device with unstable plate on $\mathrm{x}$ axis, (b) Optimal design for axial stability of the diamagnetic plate.

TABLE V: UNSTABLE DESIGN PARAMETERS AND OPTIMIZATION RESULTS

\begin{tabular}{|l|l|l|l|l|}
\hline \multicolumn{1}{|c|}{ Name } & \multicolumn{1}{c|}{ Designation } & \multicolumn{1}{c|}{ Unstable design } & \multicolumn{1}{c|}{ Optimal design } & \multicolumn{1}{c|}{ unity } \\
\hline gap & Distance between two magnets & 1.4 & 6.0075 & $\mu \mathrm{m}$ \\
\hline Magheight & Magnets height & 1.26552 & 4.7046 & $\mu \mathrm{m}$ \\
\hline Magsize & Magnets squared base size & 2.40428 & 11.0468 & $\mu \mathrm{m}$ \\
\hline diaWeight & Weight of sphere & 0.14561 & 0.14561 & $\mathrm{pN}$ \\
\hline diaArch & Archimedes force & 0.13868 & 0.13868 & $\mathrm{pN}$ \\
\hline magFZ & Vertical magnetic force & 0.007 & 0.00697 & $\mathrm{pN}$ \\
\hline FtotZ & Balance of vertical forces & $0.000066(\sim 0 \mathrm{~N})$ & $0.000037(\sim 0 \mathrm{~N})$ & $\mathrm{pN}$ \\
\hline gradFx_x & Gradient of Fx along $\mathrm{x}$ axis & $0.31019($ unstable) & $-2.8182($ stable $)$ & $\mathrm{nN} / \mathrm{m}$ \\
\hline gradFz_z & Gradient of Fz along $\mathrm{z}$ axis & $-4.0304($ stable $)$ & $-16.684($ stable) & $\mathrm{nN} / \mathrm{m}$ \\
\hline
\end{tabular}
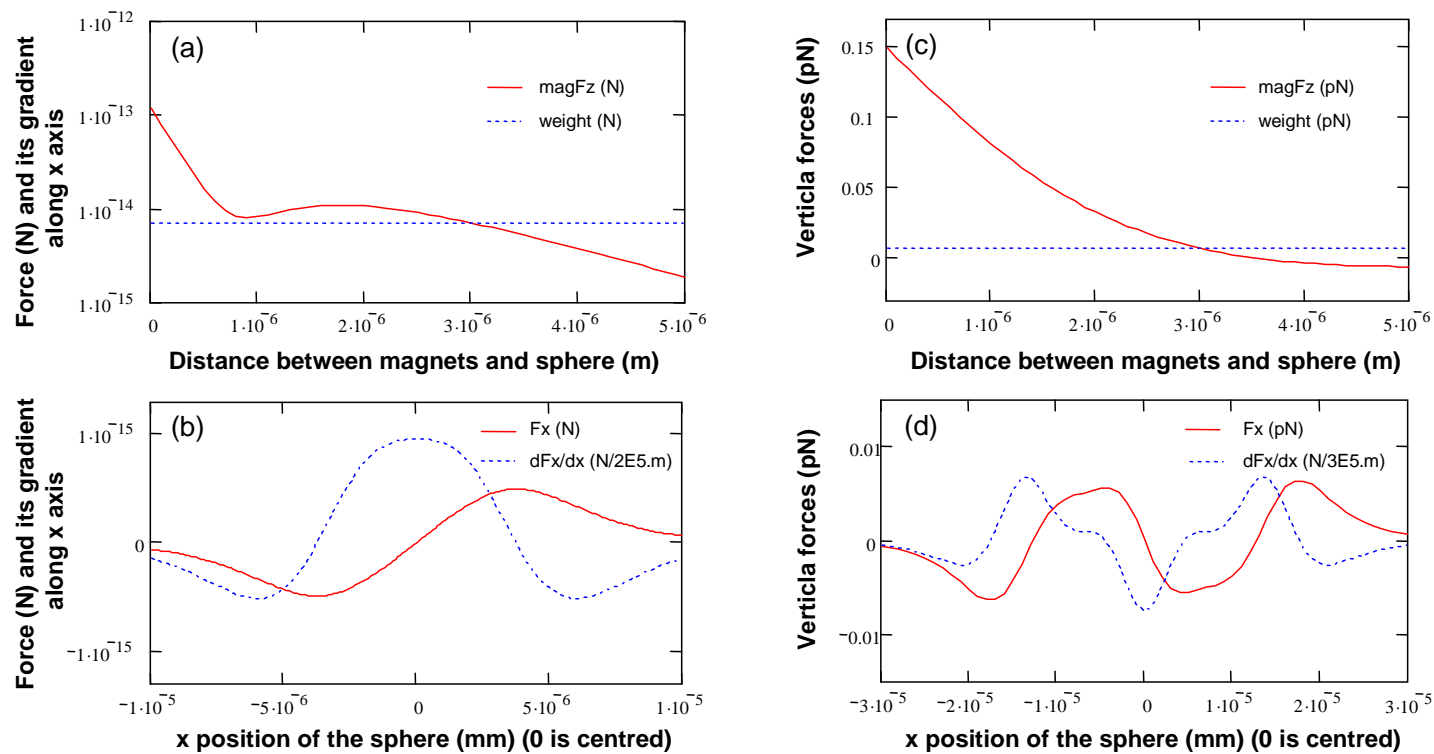

Fig. 9 - (a,b) Forces experienced by the diamagnetic plate, (a) vertical forces according to the distance between the magnets and the plate at the case of the unstable design, (b) horizontal force and its gradient according to the $\mathrm{x}$ position of the plate at the case of the unstable design, (c, d) Case of the stable design. 


\section{CONCLUSION}

We report an efficient and accurate semi-analytical modeling and optimization approach of devices based on passive diamagnetic levitation. The studied devices are composed of a levitating object and fixed part of four permanent magnets dedicated for biochemical and biomedical applications. In order to establish semi-analytical modeling of the proposed device surface approach was used to compute analytically the magnetic field induction produced by the magnets. Forces components formulas experienced by the levitating objects were also simplified to surface integral expressions. These simplifications enabled faster optimizations which were thereafter performed by a SQP algorithm. The results demonstrate a solution to perform a fast and flexible optimization of devices based on diamagnetic levitation which can be generalized to other application fields. This avoids the usual approaches which are not adequate for the optimization task in this field of applications.

\section{REFERENCES}

[1] A. Winkleman, K.L. Gudiksen, D. Ryan, G.M. Whitesides, D. Greenfield, M. Prentiss, “A magnetic trap for living cells suspended in a paramagnetic buffer," Appl. Phys. Lett., 85(12), pp 2411-2413 , 2004.

[2] N. Pamme, A. Manz, "On-chip free-flow magnetophoresis: continuous flow Separation of magnetic particles and agglomerates," Anal. Chem, 76, pp 7250-7256, December 2004

[3] H. Lee, A.M. Purdon, R.M. Westervelt, "Manipulation of biological cells using a microelectromagnet matrix,” Appl. Phys. Lett., 85(6), pp.1063-1065, June 2004.

[4] H. Watarai, M. Namba, "Capillary magnetophoresis of human blood cells and their magnetophoretic trapping in a flow system," J. Chromatogr. A, 961, pp 3-8, June 2002.

[5] C. Singh, D. Sarkar, "Practical Considerations in the Optimization of Induction Motor Design," IEE Proceedings-Electric Power Applications, vol. 139, no. 4, , pp. 365-373, July 1992.

[6] H. Chetouani, C. Jeandey, V. Haguet, H. Rostaing, C. Dieppedale, G. Reyne, "Diamagnetic levitation with permanent magnets for contactless guiding and trapping of microdroplets and particles in air and liquids", IEEE International Magnetics Conference (Intermag'06), pp. 106, San Diego, USA, Mai 8-12, 2006.

[7] H. Chetouani, C. Jeandey, V. Haguet, H. Rostaing, C. Dieppedale, G. Reyne, "Principle and Analysis of a Two-dimensional On Chip Magnetophoresis of Bioparticles for Contamination-free Biochemical Reactors", The $12^{\text {th }}$ Biennial IEEE Conference On Electromagnetic Field Computation (CEFC'06), pp. 442, Miami, USA, April 30 ${ }^{\text {th }}$-Mai $3^{\text {rd }}, 2006$.

[8] O. Chadebec, J. L. Coulomb, F. Janet, “A review of magnetostatic moments method,” IEEE Trans. Magn., vol. 42, no. 4, pp. 515-520, April 2006.

[9] B. Delinchant, F. Wurtz, E. Atienza, "Reducing Sensitivity analysis Time-Coast of Compound Model," IEEE Transactions on Magnetics, vol. 40, no. 2, pp. 1216-1219, March 2004.

[10] G. Akoun and J.-P. Yonnet, "3D analytical calculation of the forces exerted between two cuboidal magnets," IEEE Trans. Magn., vol. 20, no. 5, pp. 1962-1964, July 1984

[11] Noriyuki Hirota et al.; "Magneto-Archimedes Levitation and its Applications"; RIKEN review no 44, February 2002.

[12] M. Boukallel, E. Piat, J. Abadie, "Levitated micro-nano force sensor using diamagnetic materials," 2003 IEEE International Conference on Robotics and Automation, (ICRA'03), vol. 3, pp. 3219-3224, Taipei, Taiwan, September 14-19, 2003.

[13] D. Denis, L. Gerbaud, B. du Peloux, F. Verdiere, H.L. Rakotoarison "A Generator of Software Components dedicated to the Optimization of Engineering Systems", OIPE 2006 -September $13^{\text {th }}-15^{\text {th }}$, Sorrento, Italy 2006

[14] L .Vieux-Rochaz, D. Gamet, C. Dieppedale, B. Desloges, C. Barragatti, H. Rostaing, J. Meunier-Carus, "Electrodeposition of hard magnetic CoPtP material and integration into magnetic MEMS,” J. Micromech. Microeng.16, pp 219-224, December 2006. 